\title{
PTEN and inherited hamartoma-cancer syndromes
}

G ermline mutations in PTEN, which J encodes a dual-specificity phosphatase, have been found in two related autosomal dominant hamartoma syndromes, Cowden syndrome (CS; OMIM158350) and Bannayan-RuvalcabaRiley syndrome (BRR; OMIM153480; refs 1-6). CS is characterized by hamartomas, including gastrointestinal hamartomatous polyps, and risk of neoplasms of the thyroid, breast, uterus and skin $^{7}$. BRR has early-onset macrocephaly, hamartomatous polyposis, lipomatosis and speckled penis $^{8}$. Juvenile polyposis syndrome (JPS; OMIM174900) is an autosomal dominant inherited hamartoma syndrome characterized by the presence of gastrointestinal hamartomatous polyps and an increased risk of gastrointestinal malignancy ${ }^{9}$. The diagnosis of JPS is made only if features classic for other syndromes are not present.

Could PTEN also be the JPS gene? Three groups have found no evidence of germline PTEN mutations in 21 JPS families and 16 sporadic cases ${ }^{10-12}$ and power calculations indicated that if $10 \%$ of JPS cases were due to germline PTEN mutations, there should have been a 0.99 likelihood of detecting at least one mutation among these 37 cases. Power calculations notwithstanding, 'negative studies' always raise an uneasy spectre: were these 37 JPS cases the 37 that happen to not carry germline PTEN mutations? A fourth group strived to answer this question $^{13}$ using a broad operational definition of 'JPS'; all patients who have juvenile polyps were included, irregardless of age or presence of other features. They found three 'JPS' patients with germline PTEN mutations. An adult male
(G116) had features highly suggestive of CS, and two children (G796, G710), diagnosed at ages 3 and 14, were reported not to have manifestations of $\mathrm{CS}$ or $\mathrm{BRR}^{13}$. The penetrance of CS is well under $10 \%$ below 15 years of age ${ }^{14}$, and so while the children have JPS according to diagnostic criteria, they may develop other features of CS as they age. As phenotypic features may be shared by several hamartoma syndromes and the clinical examination is not always straightforward, specific diagnoses could be difficult. It is important to distinguish the various hamartoma syndromes, as predisposition to cancer or types of cancer may be different among them. We would like to propose that the presence of a germline PTEN mutation is a useful molecular diagnostic sign for CS or BRR (ref. 15). If a 'JPS' patient were found to harbour an occult germline PTEN mutation, then it behooves the clinician to consider CS or BRR as the diagnosis, with full implications for surveillance of the skin, thyroid, breast and uterus for cancer development. With regard to the molecular diagnosis of JPS, help is on its way ${ }^{16}$. Germline mutations in SMAD4, on 18q21.1, have been found in a subset of familial and sporadic JPS cases. SMAD4 belongs to the SMAD family of genes, which encode cytoplastic mediators in the TGF $\beta$-signalling pathway. Without much extrapolation, one can easily postulate that germline mutations in other $S M A D$ genes could account for the majority of JPS. Only time will tell whether the molecular diagnosis of the inherited hamartoma-cancer syndromes will prove more robust than that based purely on clinical criteria.

\section{Acknow ledgements}

We thank J.R. Howe V for sharing prepublication material and $O$. Gimm for critical review of the manuscript. C.E. is the Lawrence and Susan Marx Investigator in Human Cancer Genetics and a Barr Investigator.

\section{Charis Eng ${ }^{1} \&$ Monica Peacocke ${ }^{2}$}

${ }^{1}$ Translational Research Laboratory, Charles A Dana Human Cancer Genetics Unit, Department of Adult Oncology, Dana-Farber Cancer Institute, Harvard Medical School, 1 J immy Fund Way, SM 822, Boston,

Massachusetts 02115, USA. Cancer Research Campaign Human Cancer Genetics Research Group, University of Cambridge, Cambridge CB2 2QQ, UK. ${ }^{2}$ Departments of Dermatology and Medicine, Columbia University College of Physicians and Surgeons, 630 West 168th Street, VC-1526, New York, New York 10032, USA. Correspondence should be addressed to C.E. (e-mail: charis_eng@dfci.harvard.edu) or M.P. (e-mail: mp231@columbia.edu).

1. Liaw, D. et al. Nature Genet. 16, 64-67 (1997).

2. Tsou, H.C. et al. Am. J. Hum. Genet. 61, 1036-1043 (1997).

3. Nelen, M.R. et al. Hum. Mol. Genet. 6, 1383-1387 (1997).

4. Lynch, E.D. et al. Am. J. Hum. Genet. 61, 1254-1260 (1997).

5. Marsh, D.J. et al. Nature Genet. 16, 333-334 (1997).

6. Marsh, D.j. et al. Hum. Mol. Genet. 7, 507-515 (1998).

7. Eng, C. \& Parsons, R. in The Genetic Basis of Human Cancer (eds Vogelstein, B. \& Kinzler, K.W.) 519-526 (McGraw-Hill, New York, 1998).

8. Gorlin, R.J., Cohen, M.M., Condon, L.M. \& Burke, B.A. Am. J. Med. Genet. 44, 307-314 (1992).

9. Ko, F.Y., Wu, T.C. \& Hwang, B. Acta Paediatr. 36 197-202 (1995).

10. Marsh, D.J. et al. Cancer Res. 57, 5017-5021 (1997)

11. Riggins, G.J., Hamilton, S.R., Kinzler, K.W. \& Vogelstein, B. J. Neg. Obs. Genet. Oncol. (28 July, 1998) http://128.220.85.41:5002/MCGI/ SEND1 WEBUTLTY $(1378,1) / 1674326758$ (1998).

12. Howe, J.R. et al. Am. J. Hum. Genet. 62, 1129-1136 (1998).

13. Olschwang, S., Serova-Sinilnikova, O.M., Lenoir, G.M. \& Thomas, G. Nature Genet. 18, 12-14 (1998) 14. Nelen, M.R. et al. Nature Genet. 13, 114-116 (1996). 15. Eng, C. \& Ji, H. Am. J. Hum. Genet. 62, 1020-1022 (1998)

16. Howe, J.R. et al. Science 280, 1086-1088 (1998). 